\title{
An unusual presentation of X-linked adrenoleukodystrophy
}

\author{
Avinash Suryawanshi ${ }^{1,2, *}$, Timothy Middleton ${ }^{1,2,{ }^{*}}$ and Kirtan Ganda ${ }^{1,2}$ \\ 'Department of Endocrinology and Metabolism, Concord Repatriation General Hospital, Concord, \\ New South Wales 2139, Australia \\ ${ }^{2}$ Concord Clinical School, The University of Sydney, Sydney, New South Wales 2139, Australia \\ *(A Suryawanshi and T Middleton contributed equally to this case report)
}

\section{Summary}

X-linked adrenoleukodystrophy (X-ALD) is a rare genetic condition caused by mutations in the $A B C D 1$ gene that result in accumulation of very long chain fatty acids (VLCFAs) in various tissues. This leads to demyelination in the CNS and impaired steroidogenesis in the adrenal cortex and testes. A 57-year-old gentleman was referred for the assessment of bilateral gynaecomastia of 6 months duration. He had skin hyperpigmentation since 4 years of age and spastic paraparesis for the past 15 years. Physical examination findings included generalised hyperpigmentation (including skin, buccal mucosa and palmar creases), blood pressure of $90 / 60 \mathrm{mmHg}$, non-tender gynaecomastia and bilateral hypoplastic testes. Lower limb findings were those of a profoundly ataxic gait associated with significant paraparesis and sensory loss. Primary adrenal insufficiency was confirmed and investigations for gynaecomastia revealed normal testosterone with mildly elevated luteinising hormone level and normal prolactin. The combination of primary adrenal insufficiency (likely childhood onset), partial testicular failure (leading to gynaecomastia) and spastic paraparesis suggested X-ALD as a unifying diagnosis. A serum VLCFA panel was consistent with X-ALD. Subsequent genetic testing confirmed the diagnosis. Treatment with replacement doses of corticosteroid resulted in improvement in blood pressure and increased energy levels. We have reported the case of a 57-year-old man with a very late diagnosis of X-ALD manifested by childhood onset of primary adrenal insufficiency followed by paraparesis and primary hypogonadism in adulthood. Thus, X-ALD should be considered as a possibility in a patient with non-autoimmune primary adrenal insufficiency and neurological abnormalities.

\section{Learning points:}

- Adult patients with X-ALD may be misdiagnosed as having multiple sclerosis or idiopathic spastic paraparesis for many years before the correct diagnosis is identified.

- Screening for X-ALD with a VLCFA panel should be strongly considered in male children with primary adrenal insufficiency and in male adults presenting with non-autoimmune primary adrenal insufficiency.

- Confirmation of a genetic diagnosis of X-ALD can be very useful for a patient's family as genetic testing enables detection of pre-symptomatic female heterozygotes who can then be offered pre-natal testing to avoid transmission of the disease to male offsprings.

\section{Background}

This case report describes a 57-year-old man with a late diagnosis of X-linked adrenoleukodystrophy (X-ALD), who presented as a diagnostic challenge. Remarkably, the patient's primary adrenal insufficiency remained untreated for over five decades despite presenting at 4 years of age. The X-ALD was undiagnosed despite the development of spastic paraparesis two decades ago. This case would be of interest to a general endocrine audience 
because it highlights the need for clinicians to be aware of this diagnosis, especially among patients with a combination of adrenal insufficiency, spastic paraparesis and/or hypogonadism.

\section{Case presentation}

A 57-year-old male of Chinese background presented with bilateral gynaecomastia that was associated with a lack of energy, decreased libido and reduced shaving frequency. The gynaecomastia was of 6 months duration and was not associated with galactorrhoea, headache or visual disturbance. The patient was not prescribed any medications that could explain his symptoms, and there was no history of mumps orchitis, testicular trauma or testicular surgery in childhood.

His past medical history showed a 6-month hospitalization in China at 4 years of age, when he was diagnosed with Addison's disease. He was advised to take life-long corticosteroid therapy, but this did not occur; his father refused treatment due to the fear of side effects. He had noticed progressive skin hyperpigmentation since childhood.

He was diagnosed with idiopathic spastic paraparesis at the age of 53 years after presenting with a 15-year history of gait disturbance, characterised by progressively worsening stiffness of the lower limbs and ataxia, which was accompanied by urinary urgency. He reported no motor or sensory symptoms in his upper limbs. There was no history of speech disturbance, dysphagia or visual disturbance. In China, he had worked as a chemical engineer before migrating to Australia in his early fifties. He reported being a life-long non-smoker and abstained from drinking alcohol. He lived with his wife and a healthy 21-year-old son. The patient has two sisters, aged 58 and 62 years, and one brother aged 64 years who do not report any gait disturbance. The 58-year-old sister has a son aged 27 and a daughter aged 15 years, who do not have any medical problems. His other sister and brother, both have one daughter each. His parents were not reported to have significant medical problems.

Physical examination revealed generalised hyperpigmentation particularly over the knuckles, toes, palmar creases, tongue and oral mucosa (Fig. 1). His blood pressure was $90 / 60 \mathrm{mmHg}$ with no postural drop, and his heart rate was 80 beats/min. He had bilateral non-tender gynaecomastia with $\sim 4-5 \mathrm{~cm}$ of glandular tissue underneath the areola (Fig. 1). Galactorrhoea was absent. Testes were $5 \mathrm{ml}$ bilaterally. He had a male pattern hair distribution with sparse axillary and chest hair but normal pubic hair.

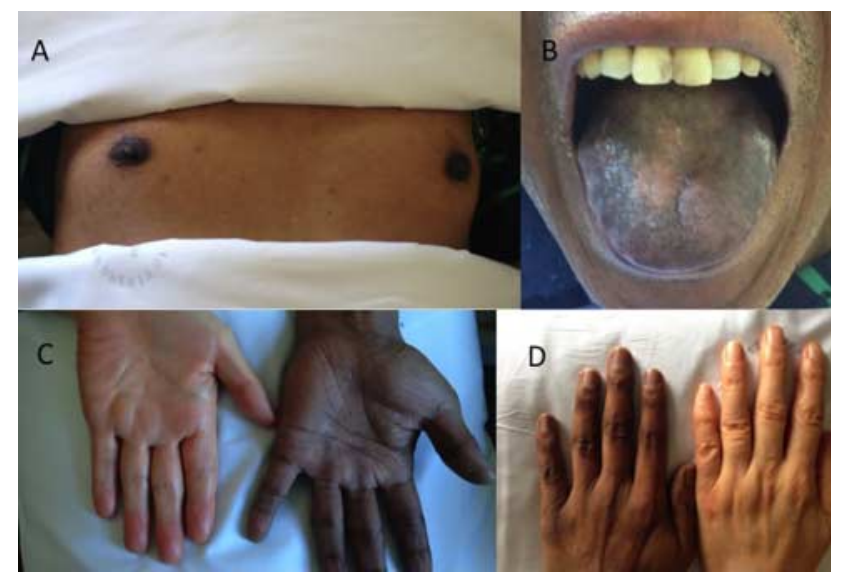

Figure 1

The patient's appearance on physical examination. (A) Bilateral non-tender gynaecomastia with $4-5 \mathrm{~cm}$ of glandular tissue under the areola.

$(B, C$ and $D)$ Generalized hyperpigmentation of the tongue, palmar creases and knuckles (Panels C and D compare the appearance of the patient's left hand with the hand of his wife who is from the same ethnic group).

Notably, he had no scalp hair loss. On neurological examination, cognition was intact; there were normal visual fields to confrontation and no cranial nerve deficits. No motor or sensory abnormalities were detected in the upper limbs. His gait was slow and ataxic, requiring the assistance of a walking cane. He had bilateral lower limb spasticity with a moderate pyramidal distribution of weakness (affecting hip flexion, knee flexion and dorsiflexion). His reflexes were exaggerated at both the knees and ankles, while plantar responses were down going. He had bilateral vibration sensory loss up to iliac crests and reduced sensation to pin prick upto the T7 sensory level.

\section{Investigation}

Initial laboratory testing (Table 1) demonstrated evidence of primary adrenal insufficiency with low morning $(0830 \mathrm{~h})$ cortisol associated with highly elevated adrenocorticotropic hormone (ACTH) levels. Adrenal 21-hydroxylase auto-antibodies were negative. Morning testosterone level was within the reference range with an elevated sex hormone binding globulin level, a slightly elevated luteinising hormone and a normal follicle stimulating hormone level. MRI of the brain and spinal cord performed 4 years prior for the assessment of spastic paraparesis was unremarkable.

Given the combination of primary adrenal insufficiency, spastic paraparesis and features of hypogonadism, a serum very long chain fatty acids (VLCFAs) panel was obtained and confirmed the elevated VLCFA levels 
Table 1 Investigations before and after commencement of hydrocortisone.

\begin{tabular}{|c|c|c|c|}
\hline $\begin{array}{l}\text { Investigational } \\
\text { parameter }\end{array}$ & $\begin{array}{c}\text { Pre- } \\
\text { diagnosis }\end{array}$ & $\begin{array}{c}\text { Post- } \\
\text { hydro- } \\
\text { cortisone }\end{array}$ & $\begin{array}{c}\text { Reference } \\
\text { range }\end{array}$ \\
\hline Morning cortisol (nmol/l) & 72 & 420 & $200-600$ \\
\hline ACTH $(\mathrm{pmol} / \mathrm{l})$ & 1118 & 73 & $<10$ \\
\hline Sodium (mmol/l) & 129 & 136 & $135-145$ \\
\hline Potassium (mmol/l) & 4.6 & 4.6 & $3.5-5.0$ \\
\hline Chloride $(\mathrm{mmol} / \mathrm{l})$ & 95 & 97 & 97-109 \\
\hline Bicarbonate (mmol/l) & 23 & 29 & $24-32$ \\
\hline Creatinine $(\mu \mathrm{mol} / \mathrm{l})$ & 80 & 65 & $70-110$ \\
\hline $\mathrm{TSH}(\mathrm{mIU} / \mathrm{l})$ & 5.9 & 2.8 & $0.3-4.5$ \\
\hline FT4 (pmol/l) & 14.7 & 12.4 & $12.0-25.0$ \\
\hline Renin activity (fmol/l per s) & - & $>5000$ & $130-2350$ \\
\hline Aldosterone $(\mathrm{pmol} / \mathrm{l})$ & - & $<40$ & $80-1040$ \\
\hline Prolactin & 28.3 & 8.5 & $4-15$ \\
\hline Testosterone (nmol/l) & 19 & 20 & $9-31$ \\
\hline LH (IU/I) & 10.4 & 14.0 & $1.7-8.6$ \\
\hline FSH (IU/I) & 14.9 & 8.7 & $1.5-12.4$ \\
\hline SHBG $(\mathrm{nmol} / \mathrm{l})$ & 85.7 & 66.5 & $19.0-83.0$ \\
\hline Estradiol (pmol/l) & 121 & - & $<206$ \\
\hline \multicolumn{4}{|l|}{ VLCFA panel } \\
\hline Hexacosonate & 8.31 & - & $0.33-1.13$ \\
\hline $\mathrm{C} 26: \mathrm{C} 22$ & 0.098 & - & $\begin{array}{r}0.007- \\
0.023\end{array}$ \\
\hline C24:C22 & 1.35 & - & $0.60-1.02$ \\
\hline
\end{tabular}

(Table 1) consistent with a peroxisomal biogenesis or betaoxidation defect. The diagnosis of X-ALD was then confirmed by gene testing with a hemizygous mutation in the $A B C D 1$ gene (c.1534G $>\mathrm{A}$ (p.Gly512Ser)).

\section{Treatment}

The patient was started on hydrocortisone replacement at a dose of $10 \mathrm{mg}$ in the morning and $4 \mathrm{mg}$ in the afternoon, with good effect. He refused to take fludrocortisone therapy due to the fear of side effects.

\section{Outcome and follow-up}

Following glucocorticoid treatment, his energy levels and blood pressure both improved significantly. He reported mild improvement in gynaecomastia and skin hyperpigmentation, but there was no change in gait disturbance, urinary urgency or lower limb weakness. His clinical improvement was accompanied by improved biochemical parameters (Table 1).

\section{Discussion}

This case report illustrates the late diagnosis of X-ALD in a 57 -year-old male despite at least 15 years of neurological symptoms on a background of primary adrenal insufficiency since childhood. The case can be considered unique in several respects.

First, the mode of presentation in this case is noteworthy due to the diagnostic delay and the fact that neurological decline occurred only in his forties. Recent literature points out that the usual presentation of X-ALD consists of primary adrenal failure in childhood, followed by slowly progressive spastic paraparesis (1). This can be accompanied by hypogonadism in the third or fourth decade. Our patient was diagnosed with adrenal failure at 4 years of age and had neurological signs for at least 15 years prior to his presentation with signs of hypogonadism. The diagnosis of ALD was not made until the relatively advanced age of 57 years.

Secondly and remarkably, this patient survived for over 50 years despite untreated hypoadrenalism. We speculate that ALD in this patient did not cause complete destruction of the adrenal glands and consequently, the patient developed relative as opposed to absolute adrenal insufficiency.

Thirdly, the absence of detectable pathology on MRI of this patient's spine despite significant clinical neurological abnormalities is noteworthy. In cerebral X-ALD, typically occipito-parietal white matter changes are the early manifestations on MRI although rarely some patients do have a normal MRI. Patients with a normal MRI may show signal changes on proton MR spectroscopy $(2,3)$. In patients with myelopathy without clinical cerebral involvement, as in our patient, it is not unusual to have a normal MRI spine, but subtle cord atrophy may be noted on MRI and is typically difficult to evaluate.

Fourthly, there was a discrepancy between the clinical and the biochemical findings with respect to hypogonadism. Despite the clinical findings of testicular atrophy and gynaecomastia, this patient's total testosterone level was firmly within the reference range. It has been proposed that decreased 5 alpha-reductase isoform 2 expression in ALD results in a reduced rate of conversion of testosterone to the more active dihydrotestosterone (4). Intuitively, one may expect that reducing the available amount of the more active dihydrotestosterone could result in hypogonadal signs and symptoms. In keeping with the findings in our patient, Assies et al. (5) demonstrated that plasma testosterone levels were generally in the reference range despite signs of clinical hypogonadism in a retrospective study of 26 men with X-ALD.

Fifthly, it is noteworthy that this patient did not develop scalp hair loss, which contrasts with the high frequency of this finding by Konig et al. (6)Two patterns of 
hair loss have been described amongst patients with $\mathrm{X}$-ALD: the first being early onset androgenetic alopecia despite hypogonadism in these patients, the second pattern is that of a diffuse reduction in scalp hair, although occasionally, there is patchy hair loss. The pathogenesis of hair loss in X-ALD has not been elucidated with one proposed mechanism being the accumulation of VLCFAs in the hair follicle.

An important lesson from this case report is that adult patients with X-ALD are often misdiagnosed as having multiple sclerosis or idiopathic spastic paraparesis for years before arriving at the correct diagnosis.

Furthermore, ALD is not an infrequent cause of idiopathic adrenal insufficiency. Consequently, screening with a VLCFA panel should be considered in male patients presenting with non-autoimmune primary adrenal insufficiency (7).

With rapid advances in the field of genetics, genetic testing of family members of patients with X-ALD is of particular importance as pre-symptomatic female heterozygotes can be offered pre-natal testing to avoid transmission of the disease to male offspring. Furthermore, confirming pre-symptomatic or mildly symptomatic female heterozygotes is important as these individuals would benefit from an early understanding of the diagnosis for prognostication and initiation of symptomatic treatment for pain, spasticity and/or sphincter disturbance. Unfortunately, despite our best efforts and referral for genetic counselling, our patient's two sisters refused to take genetic testing.

In summary, this case illustrates the late diagnosis of $\mathrm{X}$-ALD in a 57-year-old man with at least 15 years of spastic paraparesis, clinical features of adrenal insufficiency since childhood and recent onset hypogonadism.

\section{Declaration of interest}

The authors declare that there is no conflict of interest that could be perceived as prejudicing the impartiality of the research reported.
Funding

This research did not receive any specific grant from any funding agency in the public, commercial or not-for-profit sector.

\section{Patient consent}

Written informed consent has been obtained from the patient for publication of the submitted article and accompanying images.

\section{Author contribution statement}

A Suryawanshi, $\mathrm{T}$ Middleton are the joint first authors and are responsible for the initial manuscript draft as well as formatting for publication. $\mathrm{K}$ Ganda is the patient's physician, provided oversight of the reported case, made amendments to the manuscript and submitted the final version.

\section{References}

1 Engelen M, Kemp S \& Poll-The BT 2014 X-linked adrenoleukodystrophy: pathogenesis and treatment. Current Neurology and Neuroscience Reports 14 486. (doi:10.1007/s11910-014-0486-0)

2 Eichler F, Mahmood A, Loes D, Bezman L, Lin D, Moser HW \& Raymond GV 2007 Magnetic resonance imaging detection of lesion progression in adult patients with X-linked adrenoleukodystrophy. Archives of Neurology 64 659-664. (doi:10.1001/archneur.64.5.659)

3 Kumar AJ, Kohler W, Kruse B, Naidu S, Bergin A, Edwin D \& Moser HW 1995 MR findings in adult-onset adrenoleukodystrophy. AJNR. American Journal of Neuroradiology 16 1227-1237.

4 Petroni A, Cappa M, Blasevich M, Solinas M \& Uziel G 2004 New findings on X-linked Adrenoleukodystrophy: $5 \alpha$-reductase isoform 2 relative gene expression is modified in affected fibroblasts. Neuroscience Letters 367 269-272. (doi:10.1016/j.neulet.2004.05.115)

5 Assies J, Gooren LJ, Van Geel B \& Barth PG 1997 Signs of testicular insufficiency in adrenomyeloneuropathy and neurologically asymptomatic X-linked adrenoleukodystrophy: a retrospective study. International Journal of Andrology 20 315-321. (doi:10.1046/j.1365-2605. 1997.00066.x)

6 Konig A, Happle R, Tchitcherina E, Schaefer JR, Sokolowski P, Kohler W \& Hoffmann R 2000 An X-linked gene involved in androgenetic alopecia: a lesson to be learned from adrenoleukodystrophy. Dermatology 200 213-218. (doi:10.1159/000018385)

7 Horn MA, Erichsen MM, Wolff AS, Mansson JE, Husebye ES, Tallaksen CM \& Skjeldal OH 2013 Screening for X-linked adrenoleukodystrophy among adult men with Addison's disease. Clinical Endocrinology 79 316-320. (doi:10.1111/cen.12159)

Received in final form 19 October 2015

Accepted 3 November 2015 